\title{
Teoria e Prática em Avaliação Psicológica
}

\author{
João Lucas Dias-Viana ${ }^{1}$ \\ Universidade São Francisco, Itatiba-SP, Brasil
}

Esta resenha propõe-se a apresentar o livro "Avaliação Psicológica: Aspectos teóricos e práticos", organizado pelas pesquisadoras e professoras Manuela Ramos Caldas Lins e Juliane Callegaro Borsa. A obra é composta por textos de autoria de diversos pesquisadores brasileiros que buscam discutir a teoria e a prática em Avaliação Psicológica (AP). O livro possui 477 páginas, 31 capítulos e foi organizado em três partes. $\mathrm{Na}$ primeira parte (cap. 1 a 14), os autores abordam aspectos teóricos da AP, qualidades psicométricas dos instrumentos, questões éticas e de formação em AP, elaboração de documentos e competências profissionais. Questões relacionadas à avaliação de crianças e adolescentes, adultos, idosos e de pessoas com deficiência visual são abordadas na segunda parte do livro (cap. 15 a 22). A utilização da avaliação psicológica nos contextos clínico, do trânsito, do esporte, forense, hospitalar, da orientação profissional e do psicodiagnóstico é discutida na terceira parte (cap. 23 a 30).

No primeiro capítulo, "A diferenciação entre avaliação psicológica e testagem psicológica: questões emergentes", Josemberg Moura de Andrade e Hemerson Fillipy Silva Sales apresentam as diferenças entre testagem e avaliação psicológica, destacando que esta é um processo que envolve diversas técnicas e métodos, não restritas ao uso exclusivo de testes. Nessa mesma perspectiva, "Avaliação Psicológica: o papel da observação e da entrevista", segundo capítulo, Maria Lucia Tillet Nunes, Luciana Lorenço e Rita de Cássia Teixeira apresentam e discutem as técnicas de observação e entrevista, destacando a sua importância na coleta de dados em um processo avaliativo.

"Aspectos Históricos da testagem psicológica: contexto internacional e nacional" é o terceiro capítulo da obra. José Maurício Haas Bueno e Mirela Dantas Ricarte apresentam a Psicometria e sua importância para o desenvolvimento dos primeiros testes psicológicos e seus respectivos usos, o surgimento da testagem psicológica no Brasil e as atuais contribuições da Teoria de Resposta ao Item (TRI) no desenvolvimento de instrumentos. Discutem, ainda, sobre a formação em AP e o uso de métodos estatísticos na construção e validação de instrumentos. No quarto capítulo, "Tipos de testes: características e aplicabilidade”, Adriana Jung Serafini, Carine Budzyn e Tainá Ludmila Fonseca apresentam ao leitor a definição de teste psicológico, as características técnicas necessárias que o teste deve possuir, apresentam a definição de instrumentos psicométricos e projetivos, e a aplicabilidade dos testes em diversos contextos de atuação profissional.

No quinto capítulo, intitulado "Instrumentos psicológicos informatizados”, Caroline Reppold e Léia Gurgel realizam um apanhado histórico da testagem informatizada, sua construção, desenvolvimento e as vantagens e desvantagens na utilização desse tipo de avaliação. A autora destaca a necessidade de estudos de evidências de validade para testes informatizados, por ser Avaliação Psicológica Informatizada uma tendência para o futuro.

No sexto e sétimo capítulo, "Critérios para a escolha de testes psicológicos" (Lucas de Francisco Carvalho \& Rodolfo Ambiel) e "Competências e cuidados para a administração da Avaliação Psicológica e dos testes Psicológicos" (Monaliza Muniz), os autores elucidam questões fundamentais para a utilização do teste psicológico numa AP, a saber, construto, público-alvo, natureza e formato do teste, propriedades psicométricas. Além disso, destacam a importância de o psicólogo ter domínio do instrumento que utiliza para uma prática eficaz e as competências teóricas, técnicas e éticas que o profissional deve possuir para realizar AP.

Em "Validade e precisão de instrumentos de avaliação psicológica" (Rodolfo Ambiel \& Lucas de Francisco Carvalho) e "Padronização e interpretação dos resultados" (Fabiano Koich Miguel), o leitor é apresentado às qualidades psicométricas que os instrumentos devem possuir. Os objetivos principais desses capítulos são: apresentar as definições desses conceitos, quais os métodos utilizados para obtenção das qualidades psicométricas

${ }^{1}$ Endereço para correspondência: Rua Waldemar César da Silveira, 105, Jardim Cura D'ars, 13045-510, Campinas, SP. 
e a importância destas no trabalho do psicólogo quanto à compreensão e interpretação dos resultados obtidos nos testes psicológicos.

No décimo capítulo, "A importância da Psicometria na Avaliação Psicológica", Hudson Golino apresenta a história da AP, a psicometria e a teoria da medida, as fases epistemológicas dos processos avaliativos, o conceito de validade estrutural e os cinco modelos de estrutura (modelo reflexivo unidimensional, de traços correlacionados, hierárquico e bifatorial). $\mathrm{O}$ autor encerra o capítulo com a demonstração de um caso, no qual foi realizada análise da estrutura de um instrumento e concluiu-se que o teste era utilizado de forma errada.

Em "Critérios de cientificidade dos métodos projetivos", Lucila Cardoso e Anna Elisa Villemor-Amaral apresentam o conceito de métodos projetivos e a relevância deles na investigação do funcionamento da personalidade humana. As autoras debatem acerca da cientificidade dos métodos, que receberam severas críticas quanto à falta de cientificidade e apresentam pesquisas brasileiras que evidenciam as qualidades psicométricas dos testes Zulliger, Pfister, HTP e CAT-A.

No décimo segundo capítulo, "Documentos decorrentes de Avaliação Psicológica”, Vivian de Medeiros Lago apresenta ao leitor os modelos de atestado, declaração, laudo e parecer, apresentando a estrutura de cada um, suas finalidades e os princípios norteadores da elaboração documental. A autora ressalta que o aprimoramento da qualidade desses documentos se dá ao longo experiência prática do psicólogo No capítulo seguinte, "Aspectos éticos na avaliação psicológica”, Fernanda Queiroz, Joyce Segabinazi e Juliane Borsa destacam importância da formação em AP para a prática profissional qualificada e refletem acerca da responsabilidade do profissional que realiza AP quanto à tomada de decisão e seus impactos sobre o sujeito avaliado.

No décimo quarto capítulo, "A formação em avaliação psicológica no Brasil", Juliane Borsa e Joice Segabinazi discutem a formação em AP, as competências que os alunos deveriam adquirir na graduação em Psicologia e a relevância de experiências práticas, não apenas disciplinas teóricas sobre o assunto. Nesse sentido, as autoras apresentam a importância da simulação para que os alunos vivenciem em situação de aprendizagem o processo de Avaliação Psicológica e adquiram maior domínio e segurança para realizarem AP na prática profissional.

Em "Avaliação de crianças e adolescentes: aspectos cognitivos", décimo quinto capítulo, Emmy Pires apresenta o conceito de cognição, os aspectos relevantes a serem observados na realização de AP com crianças e adolescentes, orientações para a entrevista clínica e anamnese, o papel da atividade lúdica, e uma lista de instrumentos que avaliam atenção, aprendizagem e memória, linguagem, funções executivas, habilidades visuoconstrutivas e processos intelectuais. No capítulo seguinte,
"Avaliação infantojuvenil: emoções, afetos e comportamentos", Paula Cassel, Alice Brunnet e Adriane Arteche abordam a relação entre emoções e comportamento, e a importância da avaliação destas na predição da trajetória do desenvolvimento de crianças e adolescentes. As autoras destacam a insuficiência de instrumentos validados para a avaliação da população infantojuvenil no Brasil.

Adiante, "Avaliação Psicológica de crianças e adolescentes com suspeita de abuso sexual", décimo sétimo capítulo, escrito por Ana Celina Albernoz, o leitor encontra informações sobre contribuições da AP em situações de suspeita de abuso sexual de menores, as dificuldades na realização desse processo, a ética profissional requerida e quais instrumentos utilizados. A autora encerra o capítulo destacando a escassez de instrumentos que atendam a essa demanda.

No décimo oitavo capítulo, "Avaliação do autismo: do rastreamento ao diagnóstico", Mariana Seize e Juliane Borsa abordam historicamente o conceito de autismo, a etiologia e epidemiologia do transtorno, os critérios e instrumentos utilizados para rastreamento e diagnóstico do autismo. As autoras destacam que o diagnóstico é complexo e os instrumentos são essenciais no processo de avaliação. No Brasil, segundo as autoras, são escassos os instrumentos que atendam a esse objetivo.

Em "Avaliação da inteligência emocional em adultos” (José Haas Bueno, Angélica Castro \& Fernanda Correia) e "Avaliação psicológica de adultos: especificidades, técnicas e contextos" (Sérgio de Oliveira \& Mônia da Silva), os autores discutem a AP de adultos e os principais objetivos do processo. Abordam a avaliação das funções cognitivas, da memória, atenção, interesse, funcionamento emocional, personalidade e sintomas psicopatológicos em adultos e os principais testes utilizados nos contextos de aplicação.

No vigésimo primeiro capítulo, "Avaliação Psicológica do idoso: aspectos cognitivos e emocionais", Candice Holderbaum e Gabriela Wagner abordam aspectos psicológicos do envelhecimento, as particularidades da AP com idosos, na qual as principais demandas por avaliação são relacionam-se à atenção, memória, ansiedade, depressão. Apresentam instrumentos brasileiros aprovados pelo Sistema de Avaliação de Testes Psicológicos (SATEPSI) e tarefas neuropsicológicas com normas para idosos, que avaliam cognição e aspectos emocionais. As autoras destacam que a demanda por avaliação de idosos é crescente, tendo em vista o aumento desta parcela da população.

"Avaliação cognitiva de pessoas com deficiência visual", vigésimo segundo capítulo, Manuela Lins, Bartholomeu Tróccoli e Luiz Pasquali apresentam o conceito de deficiência visual, que compreende pessoas cegas e com baixa visão. Os autores afirmam que alguns testes padronizados para videntes podem ser utilizados com deficientes visuais, apontando pesquisas brasileiras que tiveram esse objetivo. Por fim, apresentam pesquisas de instrumentos em desenvolvimento para avaliação da 
inteligência dessa população específica e apontam que, até o momento, a entrevista e observação são as alternativas mais viáveis.

Os capítulos 23 a 30 abordam a prática da AP inserida em diversos contextos de atuação profissional, respectivamente, Clínica (Makilin Nunes Baptista, Nelson Hauck Filho, \& Lisandra Borges), Organizacional (Daniela Pereira), Trânsito (Fabian Rueda \& Jocemara Mognon), Esporte (Daniel Bartholomeu, José Montiel, \& Afonso Machado), contexto Legal (Sonia Rovinski), Hospitalar (Beatriz Schimidt, Simone Bolze, \& Maria Crepaldi), Carreira e Psicodiagnóstico Interventivo (Marúcia Bardagi \& Mariana Nunes). Os autores contextualizam as áreas de atuação, os conhecimentos técnicos e éticos necessários à atuação, caracterizam a prática em AP nesses contextos, os principais instrumentos utilizados, as limitações de cada área, bem como as possibilidades para futuras melhorias e desenvolvimento.

Por fim, a obra apresenta discussões pertinentes quanto à Avaliação Psicológica no Brasil, abordando temas como ética, formação, competências profissionais, $\mathrm{AP}$ em contextos específicos e orientações à prática profissional. Apresenta-se como um recurso didático, de fácil compreensão e importante fonte de informações para alunos de Graduação em Psicologia, psicólogos, bem como pesquisadores do assunto, por abordar desde os conceitos centrais até orientações a prática profissional em AP.

\section{Referência}

Lins, M. R. C., \& Borsa, J. C. (Eds.). (2017). Avaliação Psicológica: Aspectos teóricos e práticos. Petrópolis, RJ: Vozes.

\section{Sobre o autor}

João Lucas Dias Viana é Psicólogo, Graduado em Psicologia pela Universidade Estadual do Ceará. Mestrando em Psicologia, com ênfase em Avaliação Psicológica, pela Universidade São Francisco. 